\title{
A Theory Based on Conversion of RGB image to Gray image
}

\author{
Tarun Kumar $\# 1$ \\ \#Assistant Professor \\ Computer Science and Engineering Department \\ Vidya College of Engineering, Meerut (U.P)
}

\author{
Karun Verma ${ }^{\# 2}$ \\ "Assistant Professor \\ Computer Science and Engineering Department \\ Thapar University, Patiala (Punjab)
}

\begin{abstract}
The use of color in image processing is motivated by two principal factors; First color is a powerful descriptor that often simplifies object identification and extraction from a scene. Second, human can discern thousands of color shades and intensities, compared to about only two dozen shades of gray. In RGB model, each color appears in its primary spectral components of red, green and blue. This model is based on Cartesian coordinate system. Images represented in RGB color model consist of three component images. One for each primary, when fed into an RGB monitor, these three images combines on the phosphor screen to produce a composite color image. The number of bits used to represent each pixel in RGB space is called the pixel depth. Consider an RGB image in which each of the red, green and blue images is an 8-bit image. Under these conditions each RGB color pixel is said to have a depth of 24 bit. MATLAB 7.0 2007b was used for the implementation of all results.
\end{abstract}

\section{Keyword}

RGB image, Gray image, MATLAB, Pixel.

\section{IMAGE TYPES}

There are three type of image, which is described below.

\subsection{Binary image}

Logical array containing only 0 s and 1 s, interpreted as black and white, respectively.

\subsection{Grayscale image}

It is also known as an intensity, gray scale, or gray level image. Array of class uint8, uint16, int16, single, or double whose pixel values specify intensity values. For single or double arrays, values range from $[0,1]$. For uint8, values range from $[0,255]$. For uint 16 , values range from $[0,65535]$. For int16, values range from [-32768, 32767].

\subsection{True color image}

It is also known as an RGB image. A true color image is an image in which each pixel is specified by three values one each for the red, blue, and green components of the pixel scalar. M by-n-by-3 array of class uint 8 , uint 16 , single, or double whose pixel values specify intensity values. For single or double arrays, values range from $[0,1]$. For uint 8 , values range from $[0,255]$. For uint 16 , values range from $[0,65535]$.

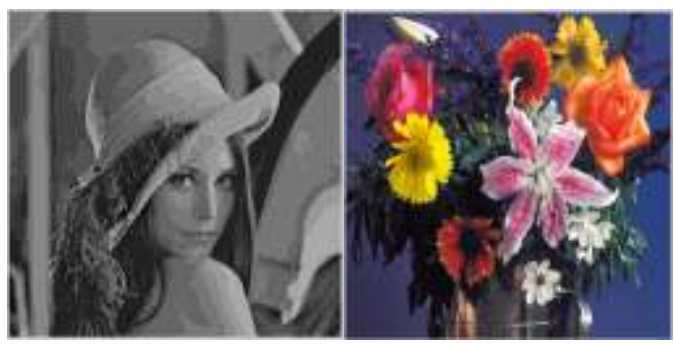

Figure1 (a): Grayscale Figure1 (b): True color

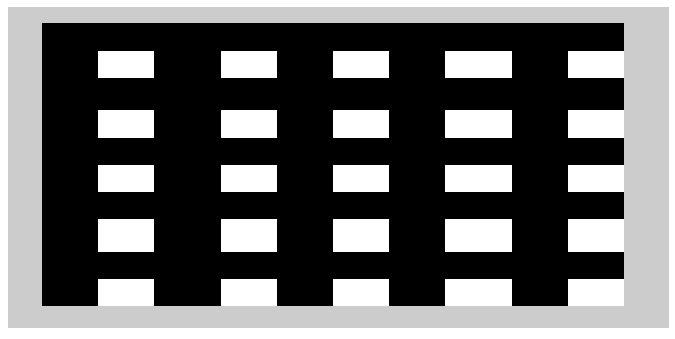

Figure 1(c): Binary

\section{GRAY SCALE IMAGES}

Image formation using sensor and other image acquisition equipment denote the brightness or intensity I of the light of an image as two dimensional continuous function $\mathrm{F}(\mathrm{x}, \mathrm{y})$ where $(\mathrm{x}$, $y$ ) denotes the spatial coordinates when only the brightness of light is considered. Sometimes three-dimensional spatial coordinate are used. Image involving only intensity are called gray scale images.

\subsection{Resolution}

Similar to one-dimensional time signal, sampling for images is done in the spatial domain, and quantization is done for the brightness values [1].

In the Sampling process [2], the domain of images is divided into $\mathrm{N}$ rows and $\mathrm{M}$ columns. The region of interaction of a row and a Coolum is known as pixel. The value assigned to each pixel is the average brightness of the regions. The position of each pixel was described by a pair of coordinates (xi, $\mathrm{xj}$ ).

The resolution of a digital signal is the number of pixel is the number of pixel presented in the number of columns $\times$ number of rows. For example, an image with a resolution of $640 \times 480$ means that it display 640 pixels on each of the 480 rows. Some other common resolution used is $800 \times 600$ and $1024 \times 728$, among other.

Resolution is one of most commonly used ways to describe the image quantity of digital camera or other optical equipment. The 
resolution of a display system or printing equipment is often expressed in number of dots per inch. For example, the resolution of a display system is 72 dots per inch (dpi) or dots per $\mathrm{cm}$.

\subsection{Gray levels}

Gray levels represent the interval number of quantization in gray scale image processing. At present, the most commonly used storage method is 8-bit storage. There are 256 gray levels in an 8 bit gray scale image, and the intensity of each pixel can have from 0 to 255, with 0 being black and 255 being white we. Another commonly used storage method is 1-bit storage. There are two gray levels, with 0 being black and 1 being white a binary image, which, is frequently used in medical images, is being referred to as binary image [3]. As binary images are easy to operate, other storage format images are often converted into binary images when they are used for enhancement or edge detection. Figure 1(a) and figure1(c) shows a typical gray scale image and a binary image, respectively.

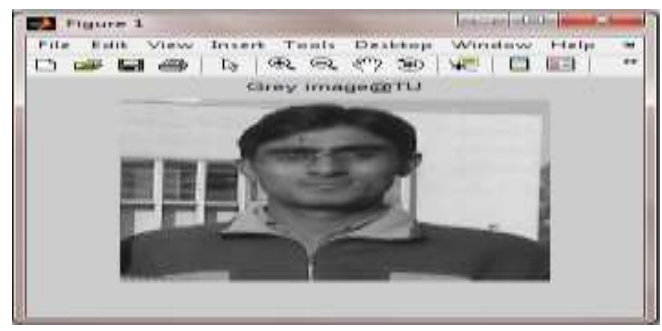

Figure 2(a): Gray image

\begin{tabular}{|c|c|c|c|c|c|c|c|c|c|c|c|}
\hline \multicolumn{8}{|c|}{ Ed Pixel Region (image tool z) } & \multicolumn{4}{|c|}{ 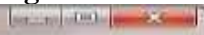 } \\
\hline File & Edit & \multicolumn{2}{|c|}{ Window } & \multicolumn{2}{|c|}{ Help } & & & & & & $\sim$ \\
\hline \multicolumn{12}{|c|}{ 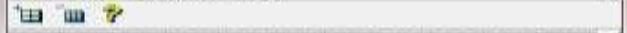 } \\
\hline 104 & 101 & 103 & 100 & 208 & 102 & 184 & 100 & 100 & 104 & 179 & \\
\hline 100 & 107 & 103 & 107 & 187 & 104 & 105 & 100 & 107 & 102 & 175 & \\
\hline 126 & 107 & 107 & 192 & 109 & 191 & 192 & 106 & 192 & 106 & 121 & \\
\hline 1 Ea & $16 \mathrm{E}$ & 169 & 160 & $16 \mathrm{~A}$ & 120 & 176 & 129 & 1 ab & 100 & 173 & \\
\hline 131 & 190 & 330 & 130 & 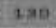 & 170 & 131 & $1 \approx 2$ & 1,23 & 134 & 150 & \\
\hline 170 & 170 & 160 & 169 & 100 & 164 & 189 & 150 & 100 & 100 & 142 & \\
\hline 207 & 207 & 200 & 207 & 206 & 202 & 197 & 104 & 193 & 103 & 100 & \\
\hline$\therefore 7$ & $1 \ldots$ & inn & $m$ & $\sin$ & tane & $\operatorname{lan}$ & $m$ & n. & 1.. & & \\
\hline & & 1,13 & & & & & & & & & \\
\hline
\end{tabular}

Fig 2(b) Pixel image of (a)

\section{RGB COLOR MODEL}

In RGB color model, each colour appears in its primary spectral componets of red,green,and blue. The colour of a pixel is made up of three components; red, renn, and blue(RGB), described by there corresponding intensities. Colour componenta are also known as colour channels or colour planes (components). In the RGB colour model, a colour image can be represented by the intensity function.

$$
\mathrm{I}_{\mathrm{RGB}}=\left(\mathrm{F}_{\mathrm{R}}, \mathrm{F}_{\mathrm{G}}, \mathrm{F}_{\mathrm{B}}\right)
$$

Where $\operatorname{FR}(x, y)$ is the intensity of the pixel $(x, y)$ in the red channel, $f G(x, y)$ is the intensitty of pixel $(x, y)$ in the greenchannel, and $\mathrm{fB}(\mathrm{x}, \mathrm{y})$ is the intensity of pixel $(\mathrm{x}, \mathrm{y})$ in the blue channel.

The intnsity of each colour channel is usually stored using eight bits, which indicates that the quantization level is 256 . That is, a pixel in a colour image requires a total storage of 24 bits. A 24 bit memory can express as $224=256 \times 256 \times 256=16777216$ distinct colours[7]. The number of colours should adequately meet the display effect of most images. Such images may be cllled true colour images, where information of each pixel is kept by using a 24-bit memory.

Figure 3 shows the images of a 24-bit colour RGB, three channels (component) and corresponding pixel information image.

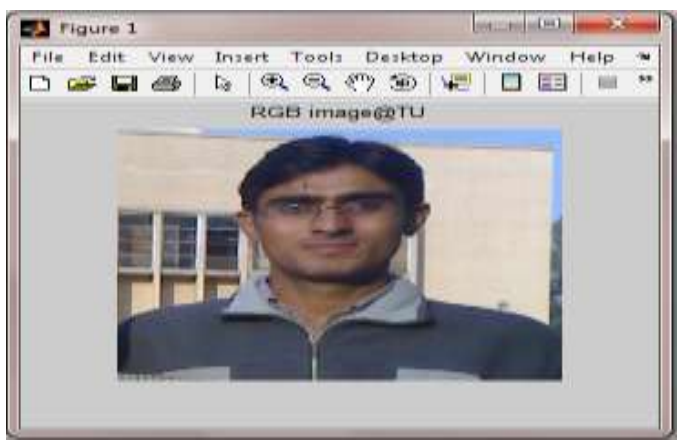

Figure 3(a): A RGB images

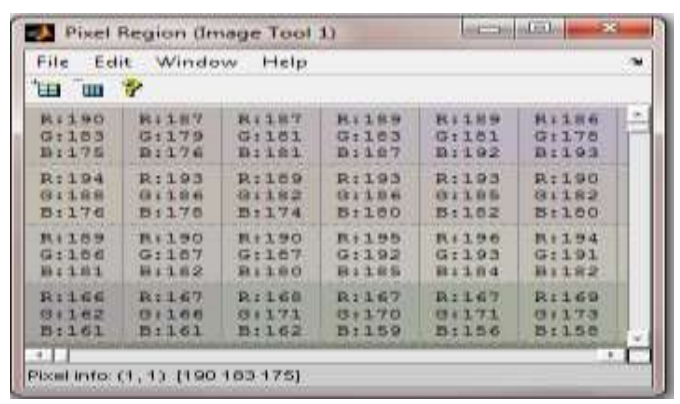

Figure 3(b): A Pixel image of (a)

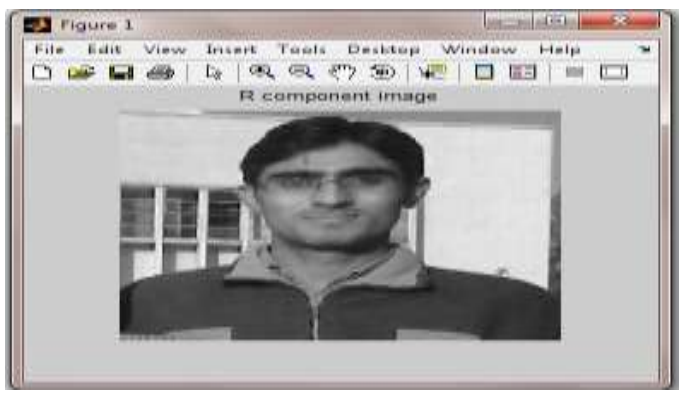

Figure 3(c): Red channel

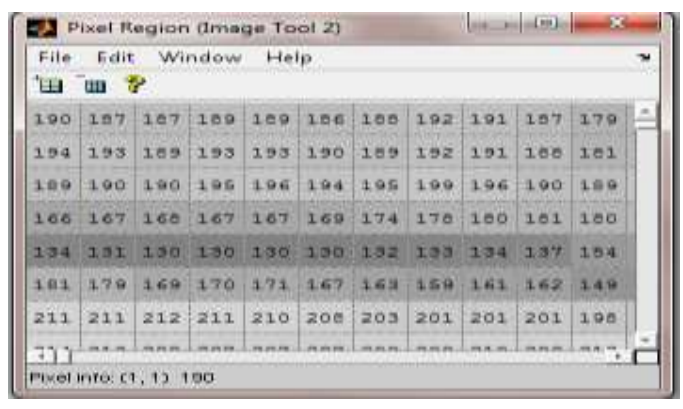

Figure 3(d): Pixel image of figure3 (c) 


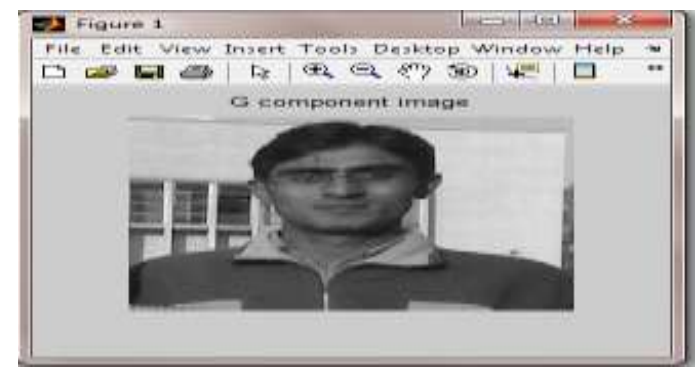

Figure 3(e): Green Channel

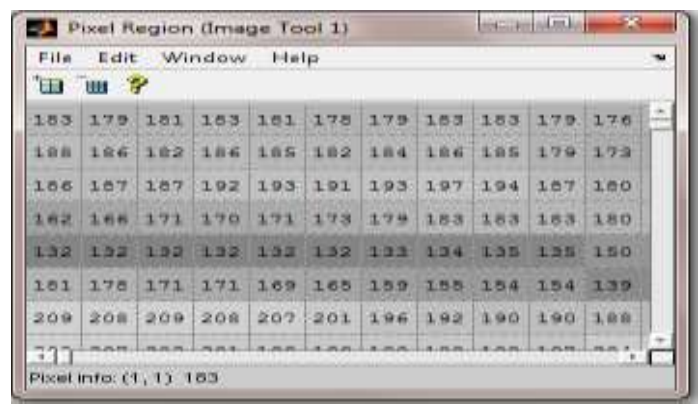

Figure 3(f): Pixel image of figure3 (e)

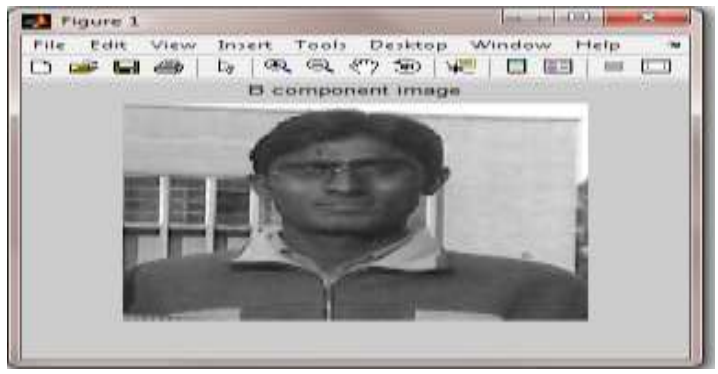

Figure 3(g): Blue channel

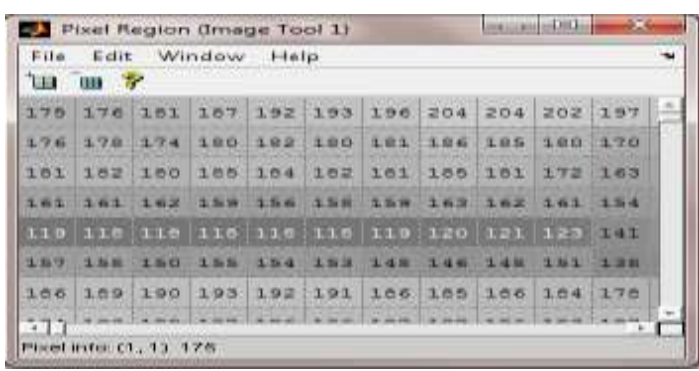

\section{Figure 3(h): Pixel image of figure(g)}

If only the brightness information is needed, color images can be transformed to gray scale images [4]. The transformation can be made by using proposed equation.

$$
\mathrm{I}_{\mathrm{y}}=0.333 \mathrm{~F}_{\mathrm{r}}+0.5 \mathrm{~F}_{\mathrm{g}}+0.1666 \mathrm{~F}_{\mathrm{b}}
$$

Where $\mathrm{Fr}, \mathrm{Fg}$ and $\mathrm{Fb}$ are the intensity of $\mathrm{R}, \mathrm{G}$ and $\mathrm{B}$ component respectively and Iy is the intensity of equivalent gray level image of RGB image.

In RGB pixel information image there are three component (R,G,B) and each component has a fix intensity 190, 183, 175( pixel info $(1,1)$ in figure $3(\mathrm{~b}))$ respectively. When RGB image converted into gray image then the intensity of pixel $(1,1)$ can be calculated by using the pixel values of RGB image in above transformation.

$$
\begin{gathered}
\mathrm{Iy}=0.333 * 190+0.5 * 183+0.1666 * 175 \\
=183.925
\end{gathered}
$$

Means in gray pixel information image the first pixel intensity is 184(1, 1). (Shown in figure 2(b))

In the similarly the intensity of second pixel $(1,2)$ (in figure 3(b) value of $\mathrm{R}, \mathrm{G}$ and $\mathrm{B}$ are 187,179 and 176 respectively) of gray level image is

$$
\begin{gathered}
\mathrm{Iy}=0.333 \times 187+0.5 \times 179+0.166 \times 176 \\
=181.15
\end{gathered}
$$

It is match with our snap shot of figure 2(b) in this we can verify all the conversation of RGB image to gray level image with transformation.

In RGB image the first pixel values for R, $\mathrm{G}$ and $\mathrm{B}$ is 190,183 , and 175 respectively. RGB image split into three images (channel) R channel, G channel and B channel. The first pixel value $(1,1)$ of these channels is $190,183,175$ respectively (as show Figure 3). Our proposed new transformation equation is given below which gives the better result than equation proposed by Karen M. Braun [6].

In this way we can calculate all the gray level by using above

\begin{tabular}{|c|c|c|c|c|c|}
\hline Pixel Number & By MATLAB & $\begin{array}{ll}\text { By } & \text { Braun } \\
\text { Method } & \end{array}$ & By Our Method & $\begin{array}{l}\text { Percentage error of } \\
\text { Braun method }\end{array}$ & $\begin{array}{l}\text { Percentage error of } \\
\text { our method }\end{array}$ \\
\hline$(1,1)$ & 184 & 184.22 & 183.925 & 0.1195 & 0.04076 \\
\hline$(1,2)$ & 181 & 181.07 & 181.159 & 0.03867 & 0.08784 \\
\hline$(1,3)$ & 183 & 182.8 & 182.9981 & 0.1092 & 0.0010 \\
\hline$(2,1)$ & 188 & 188.48 & 187.9236 & 0.2553 & 0.0406 \\
\hline$(2,2)$ & 187 & 187.22 & 186.9817 & 0.1176 & 0.00978 \\
\hline$(2,3)$ & 183 & 183.22 & 182.9254 & 0.12021 & 0.04076 \\
\hline
\end{tabular}
transformation. In Table 1 comparison of our proposed method and Braun method [6] are given and also calculate the percentage error.

Table1. Result comparisons with Braum Method 


\section{CONCLUSION}

In section 3 RGB model is shown and also show how we split RGB image into $\mathrm{R}, \mathrm{G}$, and $\mathrm{B}$ component image. Using MATLAB we convert color image into gray image then the intensity of the pixel in gray image change according to the proposed transformation equation which are explain above. Another way for proof is MATLAB snapshot. In figure 3(b) show the intensity value of $R, G$ and $B$ channel individually, using $R, G$ and $B$ value in proposed transformation equation (in section 3) calculate the intensity of gray level image then matching with the figure 2( b). In this way we explained pixel conversion of RGB image into gray image and compare proposed equation with the Braum method.

\section{REFERENCE}

[1] Gur, E., Zalevsky, Z., 2007, Single-Image Digital SuperResolution A Revised Gerchberg Papoulis Algorithm, IAENG international Journal of Computer Science, 34:2, IJCS_34_2_14.

[2] Irani, M., Peleg, S., 1991, Improving resolution by image Registration, Graphical Models and Image Processing, vol. 53, no. 3, pp.231-239.

[3] Deepa, S. N., Sivanandam, S. N., Sumathi, S., 2006, Introduction to Neural Networks using MATLAB 6.0, vol. 1, Tata McGraw-Hill.
[4] Finlayson, G. D., Qiu, G., Qiu, M., 1999, Contrast Maximizing and Brightness Preserving Color to Grayscale Image Conversion.

[5] The Math Work, 1999 the neural network and image processing toolbox, The Math Work Inc.

[6] Bala, R., Braun ,K. M., Color to grayscale conversion to maintain discriminability, Xerox Innovation Group, 800 Phillips Rd, 128-27E, Webster, NY 14580.

[7] MATLAB and Simulink for Technical Computing, URL: http://www.mathworks.com, July 2010.

[8] Pratt, W. K., 2007, "Digital Image Processing", Fourth Edition, A John Wiley \& Sons Inc. Publication.

[9] Parveen, N. R.S., Sathik, M.M., 2010, Gray Scale Conversion Of X-Ray Image, ICISA 2010, and Chennai, India.

[10] Kumar, T., Verma, K., 2010, Analysis of Soft computing Techniques for face Detection, Thesis, Thapar University, Patiala, India. 